\title{
A case of open atrial implantation of a rapid deployment valve in a patient with severe mitral annular calcification
}

Henrik Jensen Vodstrup, MD, PhD, DMSc, and Kim Terp, MD, PhD, Aarhus, Denmark

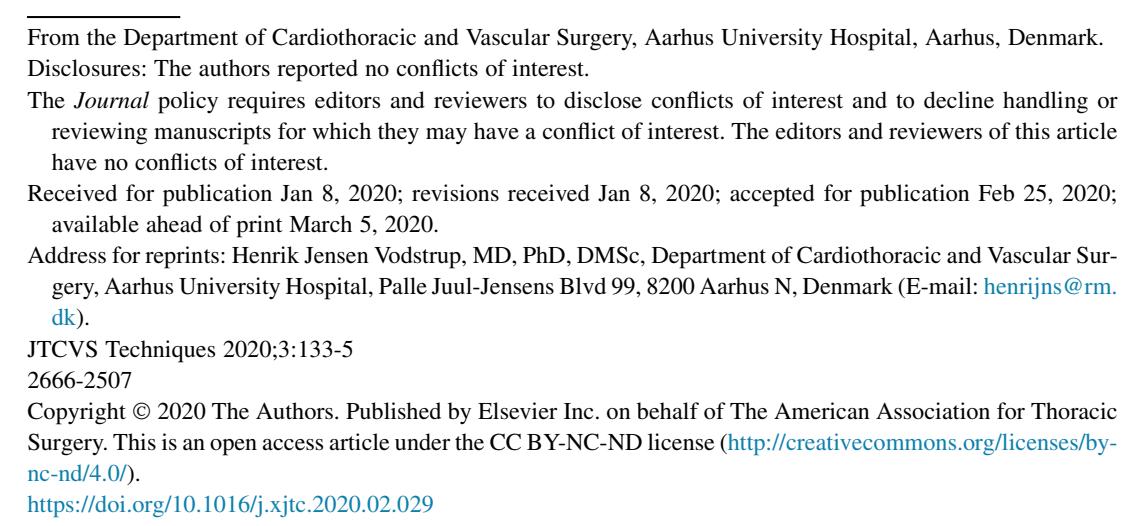

$\downarrow$ Video clip is available online.

In patients with severe mitral annular calcification (MAC), conventional surgical mitral valve implantation necessitates extensive mitral annular calcium débridement. This procedure is associated with a high risk of atrioventricular rupture, which is often fatal. To avoid removing the annular calcifications, transcatheter mitral valve replacement devices are being evaluated in clinical trials, but they are not yet widely available. ${ }^{1}$ In addition, transcatheter aortic valve replacement (TAVR) valves have been implanted in the mitral position in patients with MAC through either transapical, transseptal, or open atrial access. ${ }^{2-4}$ In this article, we present the first case of compassionate use of the Edwards Intuity Elite valve (Edwards Lifesciences, Irvine, Calif) in a patient with an open atrial case of severe MAC.

\section{PATIENT}

The patient was a 72-year-old woman in New York Heart Association functional class III with atrial fibrillation. In 2017 , because of aortic valve stenosis, the patient received a TAVR (Evolut size 26; Medtronic, Minneapolis, Minn). At that time, moderate mitral regurgitation and moderate mitral valve stenosis were present (Videos 1 and 2), but a decision was made to evaluate the effect on the patient's dyspnea from aortic valve replacement before addressing the mitral valve. Echocardiography revealed severe mitral

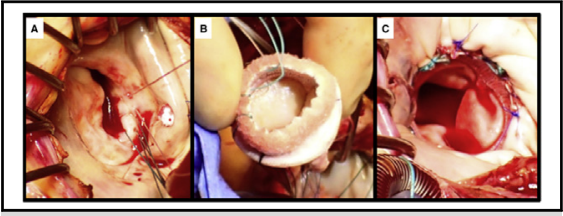

A, Calcified mitral valve annulus. B, Inverted Edwards Intuity valve. C, Implanted valve.

\section{CENTRAL MESSAGE \\ An inverted Edwards Intuity Elite rapid deployment valve was im- planted in the mitral position in a patient with severe mitral annular calcification.}

See Commentaries on pages 136 and 138.

valve stenosis (mean gradient of $15 \mathrm{~mm} \mathrm{Hg}$ ), severe mitral regurgitation, moderate pulmonary hypertension, and left ventricular ejection fraction of $60 \%$. Coronary angiogram and kidney function were normal. Computed tomographic scan showed extensive circumferential MAC. Conventional surgery with mitral annular decalcification was deemed to be associated with a very high risk of aortoventricular rupture, and we therefore planned to implant an Intuity Elite valve in an inverted fashion.

\section{SURGICAL PROCEDURE}

Sternotomy was done, and extracorporeal circulation and cardioplegic arrest were established. The left atrium was opened in the groove of Sondergaard. The mitral valve was examined, which confirmed severe MAC, and for most of the circumference it was not possible to pass needles through the annulus. This confirmed our decision to implant a rapid-deployment balloon-expandable valve in the mitral annulus. Nine 2.0 Ti-Cron sutures (Medtronic) with felt pledgets were placed through the leaflet close to the annulus. A few sutures could be placed properly in the anterior annulus. The anterior commissure was completely fused, but the posterior commissure was open. To avoid paravalvular leaks from this point, the posterior commissure was closed with sutures. To ensure maximum annular support, and because a very large angle between the aortic and mitral annulus predicted a low risk of left ventricle outflow tract (LVOT) obstruction, we did not remove the anterior 


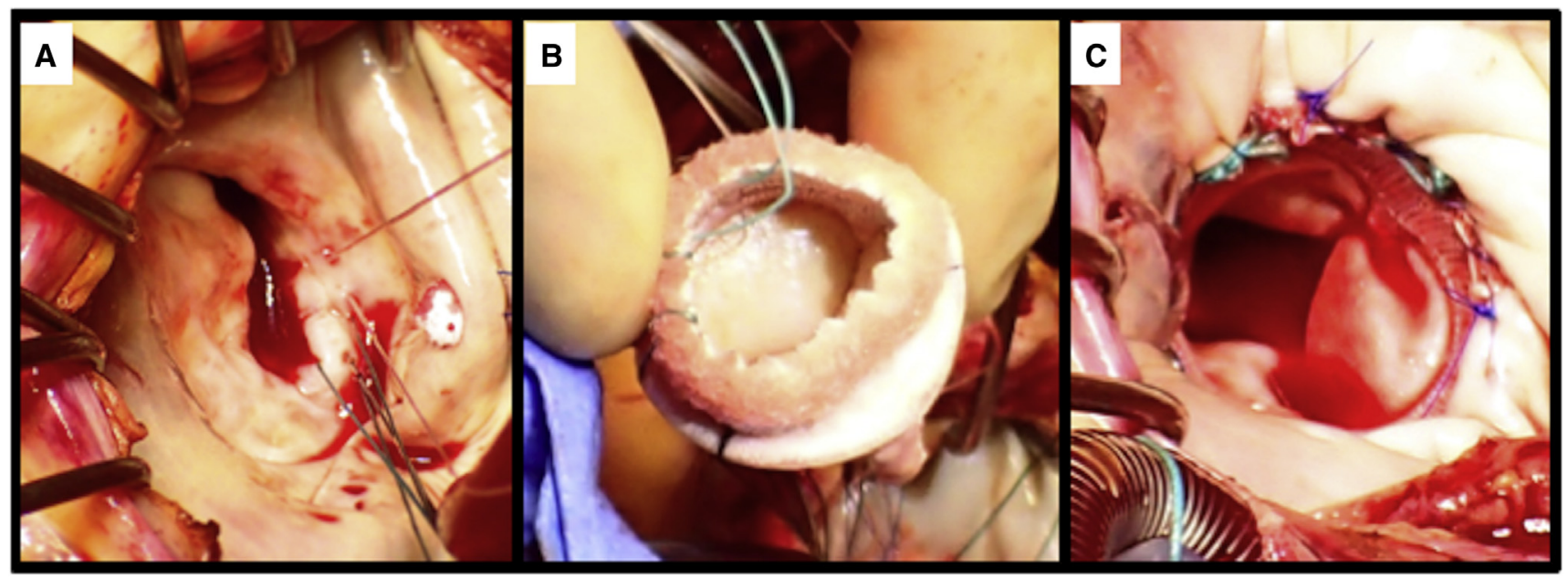

FIGURE 1. A, Calcified mitral valve annulus with sutures passed through the anterior leaflet or annulus, B, Inverted Edwards Intuity valve (Edwards Lifesciences, Irvine, Calif) with sutures passing the cloth covered stent, C, Implanted Intuity valve with running suture from stent edge to the left atrial wall.

mitral leaflet. The mitral valve annulus was predilated with a 25-mm balloon valvuloplasty catheter (Bard True Dilatation; BD Bard Medical Division, Covington, Ga) to a nominal pressure of 3 atmospheres. With Intuity valve sizers, a size 23 Edwards Intuity Elite valve was implanted in an inverted fashion, with the sewing ring on the ventricular side and the expandable stent on the atrial side of the annulus. This displaced the valve a few millimeters into the left ventricle relative to a conventional mitral valve prosthesis. The sutures were passed through the fabric-covered metal frame of the expandable stent and held in place with tourniquets. The valve stent was then expanded with the Edwards delivery system balloon, inflated to a nominal pressure of 4.5 atmospheres with an inflation device (Atrion QL 96417; Atrion Medical, Arab, Ala). This seated the valve firmly in the annulus, and the Ti-Cron sutures were tied down on the metal frame. The edge of the expanded stent on the atrial side was then sutured to the atrial wall with a 4.0 Prolene running suture (Ethicon Inc, Somerville, NJ) to avoid any paravalvular leaks. Figure 1 shows the calcified

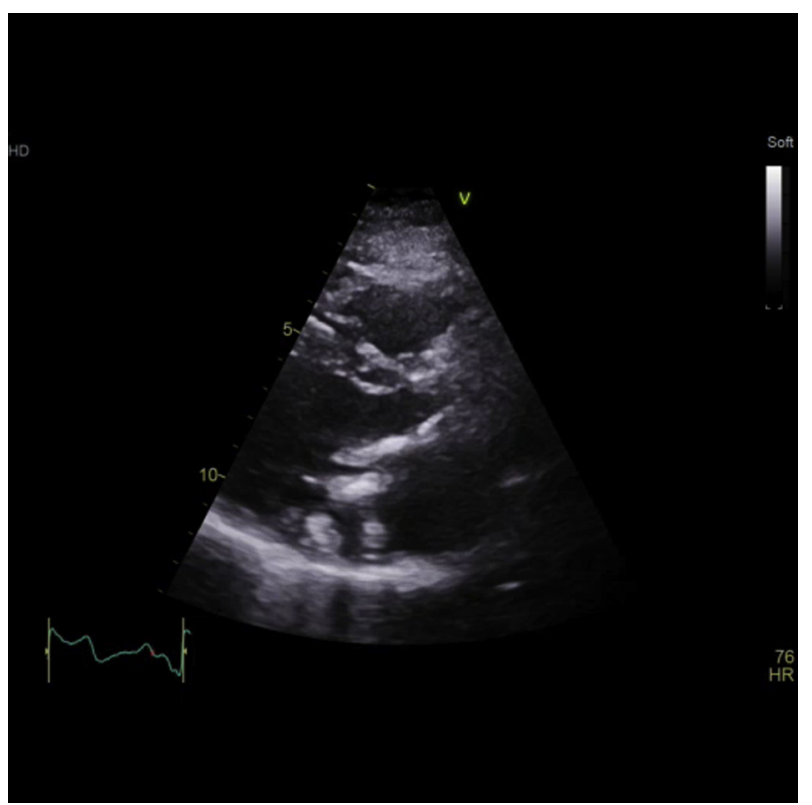

VIDEO 1. Preoperative echocardiography without color showing severe calcification and restriction of leaflet motion. Video available at: https:// www.jtcvstechniques.org/article/S2666-2507(20)30135-8/fulltext.

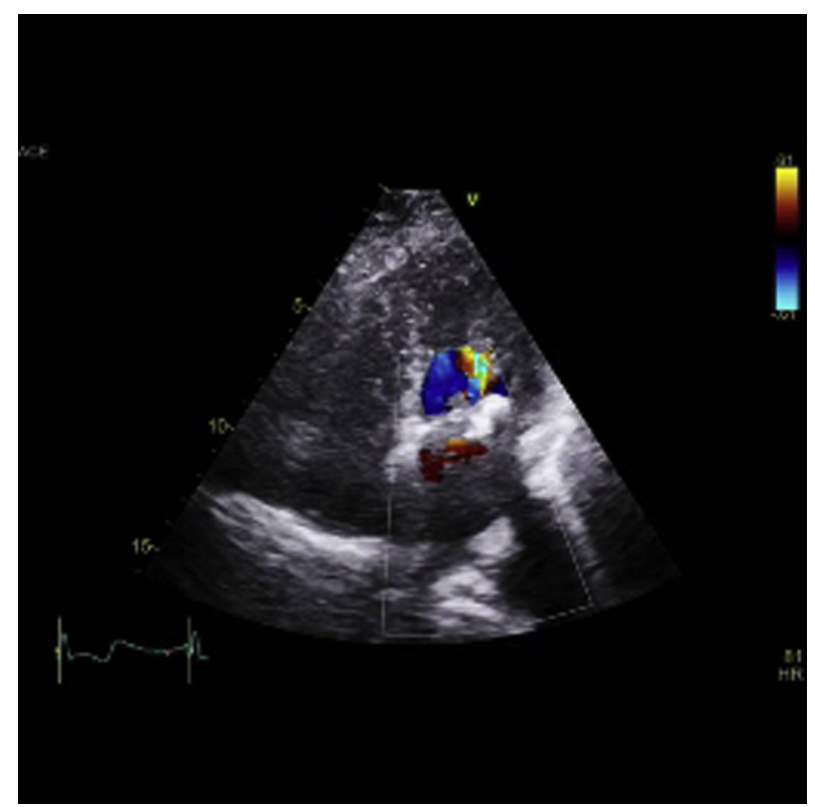

VIDEO 2. Preoperative echocardiography with color showing severe mitral regurgitation and stenosis. Video available at: https://www. jtcvstechniques.org/article/S2666-2507(20)30135-8/fulltext. 


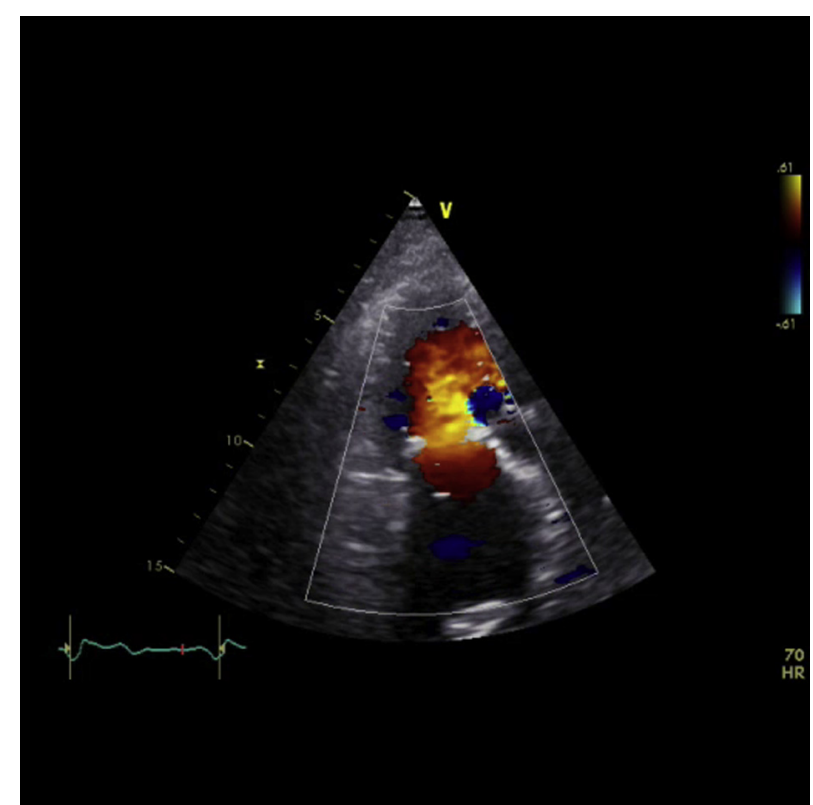

VIDEO 3. Postoperative echocardiography with color showing a wellfunctioning mitral valve prosthesis without paravalvular leaks but with some diastolic flow acceleration compatible with mild stenosis. There is also some flow acceleration in the left ventricular outflow tract compatible with a mild degree of obstruction. Video available at: https://www. jtcvstechniques.org/article/S2666-2507(20)30135-8/fulltext.

mitral valve annulus with sutures passed through the anterior leaflet, the Inverted Edwards Intuity valve with sutures passing the cloth-covered stent, and, finally, the Intuity valve after implantation and fixation with the running suture from stent edge to the left atrial wall. The atrium was closed, and the patient was weaned from extracorporeal circulation. Postoperative echocardiography showed a wellseated valve in the mitral position with a mean gradient of $5 \mathrm{~mm} \mathrm{Hg}$, with a position very similar to that of a standard mitral valve prosthesis, with only a few millimeters of displacement into the left ventricle (Video 3). The Evolut valve in the aortic position was unaffected, without regurgitation or stenosis and with a mean gradient of $8 \mathrm{~mm} \mathrm{Hg}$. The patient was transferred to the ward the next morning and had an uneventful recovery, after which she was discharged to her home 8 days after surgery. Echocardiography on the third postoperative day showed a mean mitral valve gradient of $7 \mathrm{~mm} \mathrm{Hg}$ and no paravalvular leak. Some degree of anatomic LVOT narrowing was evident on echocardiography, and the mean and peak LVOT gradients of 8 and $14 \mathrm{~mm} \mathrm{Hg}$, respectively, revealed a mild degree of LVOT obstruction. At 3 months of follow-up in the outpatient clinic, the patient was doing fine, with only mild dyspnea. The patient had a minor ischemic stroke 4 months after the operation, but she is currently in recovery and performs

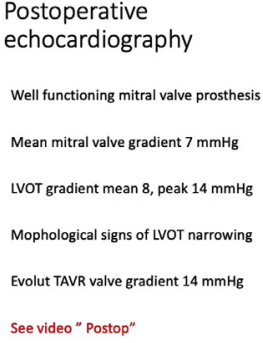

VIDEO 4. In this video, we present a case of severe mitral annular calcification treated with an inverted Edwards Intuity valve (Edwards Lifesciences, Irvine, Calif), describing our technical approach and considerations and also the lessons that we have learned from this first case. Video available at: https://www.jtcvstechniques.org/article/S2666-2507(20)30135-8/ fulltext.

normal activities of daily living. The patient provided informed consent for the publication of the study data.

\section{DISCUSSION}

To our knowledge, this is the first reported case of the use of an inverted Edwards Intuity Elite rapid deployment valve to treat severe mitral valve stenosis resulting from MAC. We chose this valve as an alternative to an inverted TAVR valve in the mitral position to avoid pushing the mitral aortic membrane toward the LVOT, with inherent risk of obstruction and displacement of the Evolut valve, to facilitate a running suture line on the atrial edge of the metal frame to prevent paravalvular leaks, and to avoid having to make adaptations on the prosthesis with felt strips. Meanwhile, unlike with TAVR, the valve cannot be dilated into the calcium but has to be able to pass through the calcified annulus. Accordingly, we could only implant a size 23 valve, which left the patient with moderate mitral stenosis. In addition, a mild degree of LVOT obstruction was present. In future patients, we plan to remove the anterior leaflet, as reported previously, ${ }^{2}$ to enable implantation of a larger valve and avoid LVOT obstruction. A video presentation of this case is available in the supplementary materials (Video 4).

\section{References}

1. Sorajja P, Moat N, Badhwar V, Walters D, Paone G, Bethea B, et al. Initial feasibility study of a new transcatheter mitral prosthesis: the first 100 patients. J Am Coll Cardiol. 2019;73:1250-60.

2. Praz F, Khalique OK, Lee R, Veeragandham R, Russell H, Guerrero M, et al Transatrial implantation of a transcatheter heart valve for severe mitral annular calcification. J Thorac Cardiovasc Surg. 2018;156:132-42.

3. Guerrero M, Urena M, Himbert D, Wang DD, Eleid M, Kodali S, et al. 1-year outcomes of transcatheter mitral valve replacement in patients with severe mitral annular calcification. J Am Coll Cardiol. 2018;71:1841-53.

4. Kassar M, Khalique OK, Pilgrim T, Reineke D, Carrel T, Windecker S, et al. Surgical transatrial implantation of transcatheter heart valves in severe mitral annular calcification. Intervent Cardiol Clin. 2019;8:313-9. 